Supporting Information for the article:

\title{
Clustering versus percolation in the assembly of colloids coated with long DNA.
}

\author{
Nienke Geerts ${ }^{\mathrm{a}}$, Tatiana Schmatko ${ }^{\mathrm{d}}$ and Erika Eiser ${ }^{\mathrm{b}, \mathrm{c}}$ \\ a. FOM Institute AMOLF, Kruislaan 407, 1098 SJ Amsterdam, The Netherlands. \\ b. HIMS Institute, University of Amsterdam, Nieuwe Achtergracht 166, 1018 WV Amsterdam. \\ c. University of Cambridge, Department of Physics, Cavendish Laboratory, Madingley Road, Cambridge \\ CB3 0HE, UK. \\ d. Institut Charles Sadron et Universite Louis Pasteur, CNRS UPR 22, 6 rue Boussingault, BP 40016, F- 67 \\ 083 Strasbourg Cédex, France.
}

Corresponding author: eiser@science.uva.nl

Figure S1: Lower resolution confocal image of clustered colloids grafted with pBeloDNA (a higher resolution, zoomed in image is shown in figure 1B), obtained after letting the sample aggregate for one month. Here several images, taken at the same focal plane, were assembled to get a better in-plane image of the percolating 3D-cluster. 


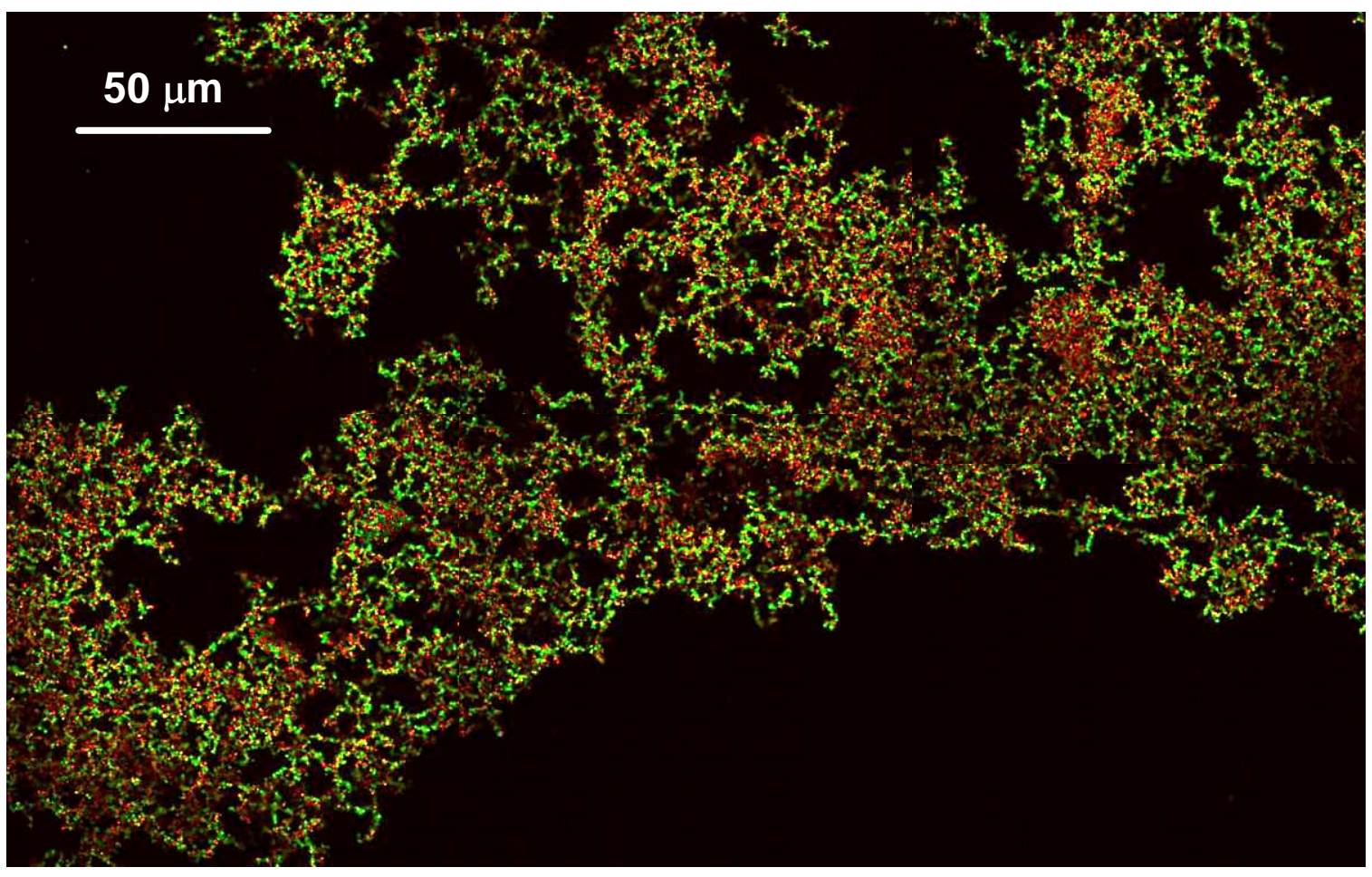

And the continuation of this cluster: 


\section{$50 \mu \mathrm{m}$}

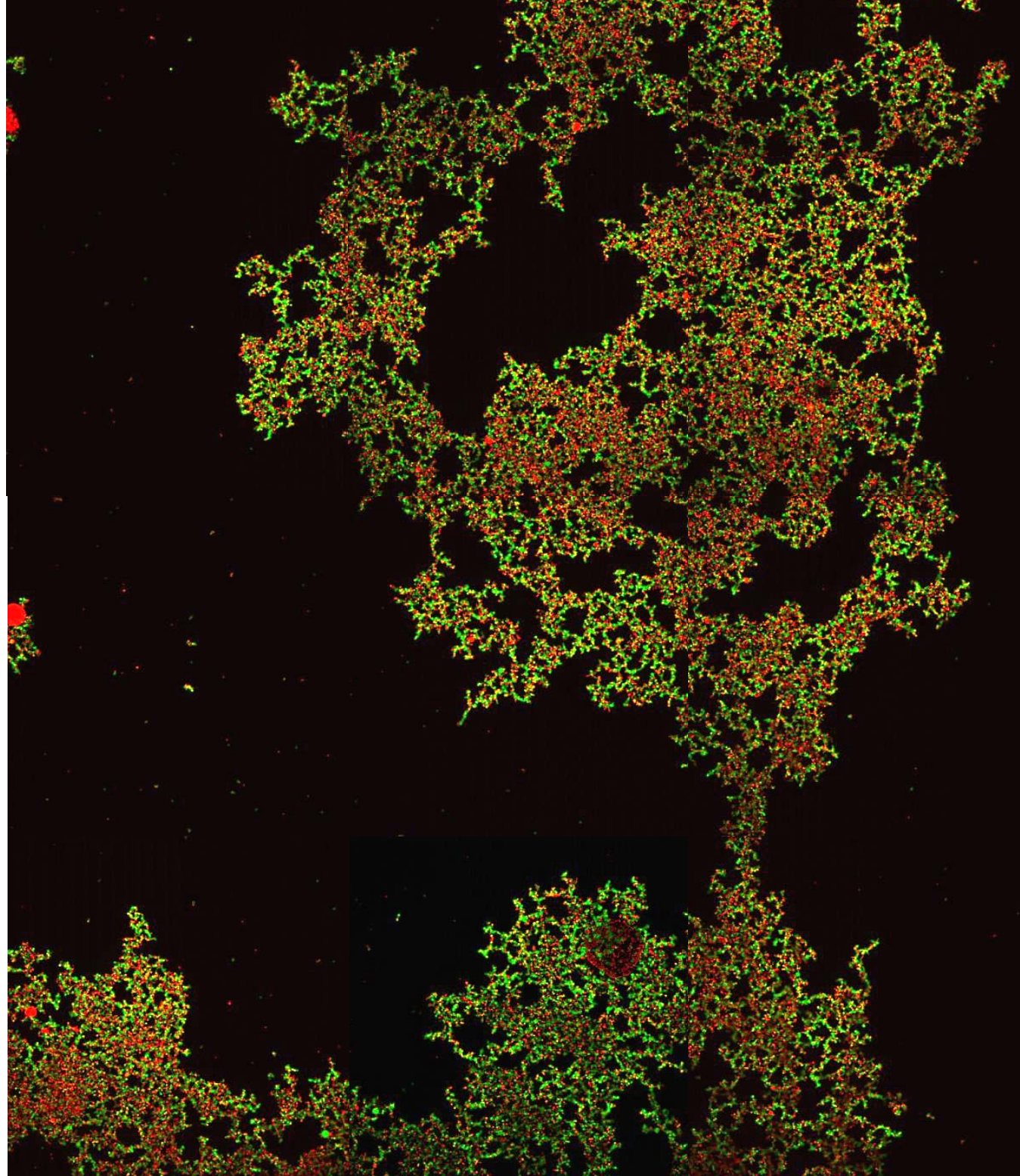


Figure S2: Left: Reconstructed 3D image of colloids with about 25 grafted pBelo-DNA arms, where on average 8 arms contain sticky cos-ends, while the remaining arms have blunt ends. Right: Cumulative graph of the cluster sizes of colloids with 8 compared to 25 sticky ends, but the same DNA-arms per bead; green: pBelo 8 stickers (2 weeks old). Orange: pBelo 25 stickers (one month old).
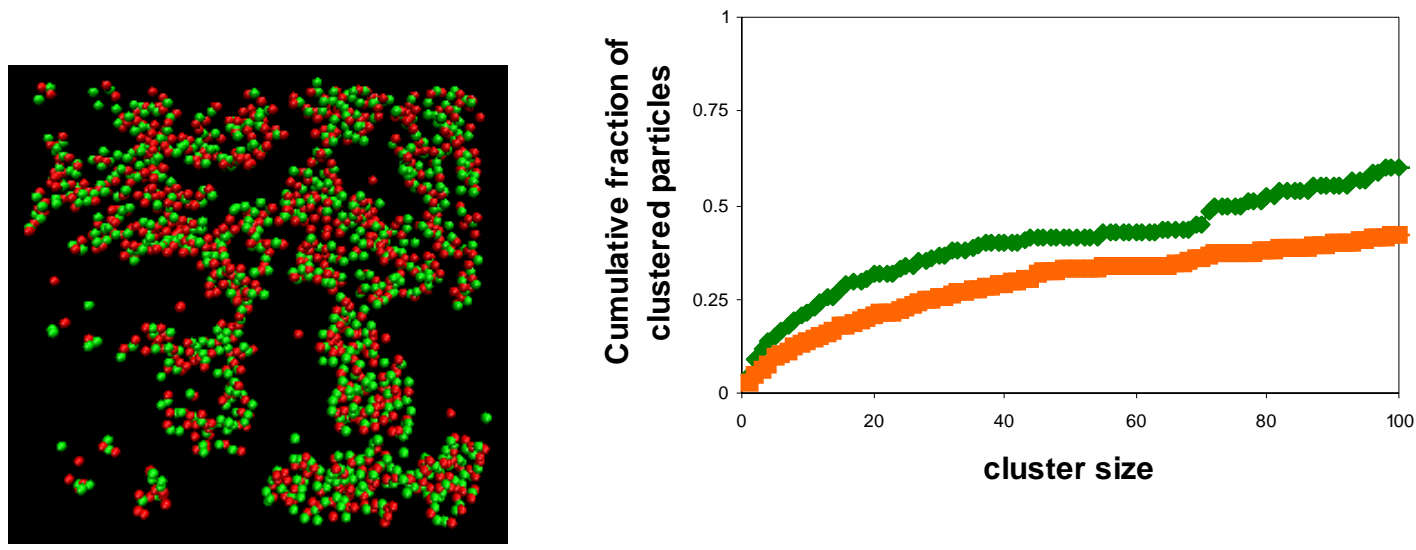